[Article]

\title{
基于团簇结构模型的 $\mathrm{Na}_{2} \mathrm{O}-\mathrm{Al}_{2} \mathrm{O}_{3}-\mathrm{SiO}_{2}$ 三元体系熔体 吉布斯混合摩尔自由能计算
}

李永康 尤静林 ${ }^{*}$ 王 建 王 敏 马 楠 魏广超

(省部共建高品质特殊钢冶金与制备国家重点实验室，上海市钢铁冶金新技术开发应用重点实验室，

上海大学材料科学与工程学院, 上海 200072)

\begin{abstract}
摘要: 以三元硅酸盐熔体团簇结构模型为基础, 选取了 $\mathrm{Na}_{2} \mathrm{O}-\mathrm{Al}_{2} \mathrm{O}_{3}-\mathrm{SiO}_{2}$ 体系不同成分的团簇结构, 采用半 经验量子化学方法 MNDO/d 分别计算该三元体系熔体中不同结构的团簇基元在 1473、1873、2000 K温度下 的熵、焓、热容和自由能等热力学数据, 计算得出不同团簇结构基元的混合自由能, 并根据统计热力学波尔 兹曼分布定律, 推导计算得出 $\mathrm{Na}_{2} \mathrm{O}-\mathrm{Al}_{2} \mathrm{O}_{3}-\mathrm{SiO}_{2}$ 三元体系各成分下的混合摩尔自由能。三元硅酸盐熔体的热 力学性质与该熔体的微观结构密切相关。
\end{abstract}

关键词: $\mathrm{Na}_{2} \mathrm{O}-\mathrm{Al}_{2} \mathrm{O}_{3}-\mathrm{SiO}_{2} ;$ 团簇结构模型; 熔体; 混合摩尔自由能

中图分类号: 0642

\section{Molar Gibbs Mixing Free Energy Calculation for a $\mathrm{Na}_{2} \mathrm{O}-\mathrm{Al}_{2} \mathrm{O}_{3}-\mathrm{SiO}_{2}$ Ternary Melt Based on the Cluster Model}

\author{
LI Yong-Kang YOU Jing-Lin* WANG Jian WANG Min MA Nan WEl Guang-Chao \\ (State Key Laboratory of Advanced Special Steel, Shanghai Key Laboratory of Advanced Ferrometallurgy, \\ School of Materials Science and Engineering, Shanghai University, Shanghai 200072, P. R. China)
}

\begin{abstract}
Based on the cluster model of molten ternary silicates, the thermodynamic properties, including entropy, enthalpy, and heat capacity of $\mathrm{Na}_{2} \mathrm{O}-\mathrm{Al}_{2} \mathrm{O}_{3}-\mathrm{SiO}_{2}$ at 1473,1873 , and $2000 \mathrm{~K}$ were calculated by the modified neglect of differential overlap (MNDO/d) semi-empirical method based on the primitive assumption of clusters in the melt. The mixing free energies of the $\mathrm{Na}_{2} \mathrm{O}-\mathrm{Al}_{2} \mathrm{O}_{3}-\mathrm{SiO}_{2}$ ternary system were derived. The mixing free energy of the $\mathrm{Na}_{2} \mathrm{O}-\mathrm{Al}_{2} \mathrm{O}_{3}-\mathrm{SiO}_{2}$ ternary system is the sum of all the cluster units that exist according to the Boltzmann distribution law. The thermodynamic properties of this ternary silicate melt depend on its microstructure.
\end{abstract}

Key Words: $\mathrm{Na}_{2} \mathrm{O}-\mathrm{Al}_{2} \mathrm{O}_{3}-\mathrm{SiO}_{2} ; \quad$ Cluster model; Melt; $\quad$ Molar mixing free energy

1 引 言

硅酸盐及含铝硅酸盐熔体的热力学性质一直
是冶金、地学、玻璃和陶瓷等领域重要的研究课 题之一。而对于硅酸盐的熔体热力学性质的研

Received: September 28, 2015; Revised: December 28, 2015; Published on Web: December 28, 2015.

*Corresponding author. Email: jlyou@163.com; Tel: +86-13331992297.

The project was supported by the Key Project of National Natural Science Foundation of China (50932005), National Natural Science Foundation of China (20973107, 40973046), Shanghai Committee of Science and Technology, China (12520709200), and CSIRO Minerals Down Under Flagship of Australia.

国家自然科学基金重点项目(50932005), 国家自然科学基金(20973107, 40973046), 上海科学技术委员会(12520709200)和澳大利亚 Flagship 的 科学与工业研究组织 CSIRO资助项目 
究, 已经有文献分别从分子模型、分子离子共存 模型、聚合模型等方面计算和讨论硅酸盐活度和 组分之间的关系, 进而利用活度求出热力学数 据 ${ }^{1-5}$ 。分子离子共存模型和聚合模型是应用比较 广泛的两个热力学模型。分子离子共存理论模型 最初是由前苏联丘意柯教授基于实验事实提出, 张鉴 ${ }^{2}$ 用此模型对二元硅酸盐熔体、三元硅酸盐熔 体和熔盐做了验证。很多考虑到硅酸盐中的聚合 反应的模型都应用到硅酸盐的热力学性质预测中 并得到后续的发展, 包括 Lin 和 Pelton ${ }^{5}$, Masson $^{6}$, Ottonello $\mathrm{o}^{7}$ Z Zaitsev 等 ${ }^{8}$ 的研究。聚合模型是假设在 给定的温度压强下各成分下的硅酸盐熔体由金属 阳离子、离子氧化物和 $\mathrm{SiO}_{4}^{4-}$ 单元均衡分布聚合而 成。关于硅酸盐聚合理论的研究已经扩展到三元 体系当中。Glibin 和 $\mathrm{King}^{9}$ 基于当前对硅酸盐熔体 结构的认识, 以 Guggenheim 的准化学模型预测了 二元体系的硅酸盐和铝硅酸盐的混合热力学函 数, 他们利用氧化物晶体、含氧分子和离子中已 有的结构和热力学数据计算模型中所需的参数, 计算结果与实验数据吻合较好。

从 Neuville ${ }^{10}$, Yang ${ }^{11}$ 和刘钦 ${ }^{12}$ 等的研究中都可 以得知铝硅酸玻璃中铝的配位数是四、五和六, 四配位铝占多数。长期以来人们一度认为玻璃继 承了熔体的结构, 因而很多学者凭借对玻璃的研 究来说明熔体结构。可是, 玻璃的结构不能等同 于熔体的结构。刘钦等 ${ }^{12}$ 通过高温拉曼光谱对铝硅 酸盐的研究认为铝在硅酸盐熔体中可以发现四配 位和六配位的结构而且主要的配位数是四, 但并 没有证据证明有五配位铝的结构存在于熔体结构 中。说明虽然很多研究指出铝硅酸盐玻璃中铝的 配位数是四、五和六, 但是铝硅酸盐熔体中只有 含四配位铝和六配位铝的结构。类似的例子可以 从锗酸盐和硼酸盐的研究中发现。锗酸盐玻璃和 晶体中锗的配位数为四和六, 但是熔体中只有四 配位锗 ${ }^{13}$; 嗍酸盐玻璃和晶体中嗍的配位数为三和 四, 但是熔体中只有三配位硼 ${ }^{14,15}$ 。

You 等 ${ }^{16,17}$ 利用高温拉曼光谱和量子化学从头 计算对硅酸盐晶体、玻璃和熔体结构进行了研 究, 并预测了硅酸盐熔体中可能存在的团簇结构 基元。除此之外, 王威和尤静林 ${ }^{18}$ 基于团簇结构模 型采用半经验方法对碱金属二元硅酸盐的热力学 性质进行了计算, 得出 100-2000 K 时的熵、焓、 吉布斯自由能及比定压热容, 并由此计算出该二
元体系的混合自由能, 并与实验数据相吻合。从 硅酸盐微观精细结构出发计算得到某成分下硅酸 盐宏观热力学性质是新的研究方法, 具有指导意 义。因此本文基于 $\mathrm{Na}_{2} \mathrm{O}-\mathrm{Al}_{2} \mathrm{O}_{3}-\mathrm{SiO}_{2}$ 三元体系熔体 团簇结构, 采用量子化学中的半经验方法, 进行 三元体系的高温热力学性质计算, 给出了该体系 的热力学性质随温度的变化规律。

\section{2 实验部分}

\section{1 铝硅酸盐熔体团簇结构模型}

团簇的选取既要考虑相关晶体的结构, 同时 也包含了熔体和玻璃体系特有的结构 ${ }^{19,20}$ 。尤静林 等 ${ }^{12,17,21}$ 采用高温拉曼光谱技术探测了硅酸盐和铝硅 酸盐类熔体, 并将熔体里的局域结构与拉曼光谱 位移建立了对应的关系, 并用量子化学从头计算 进行相关有效的验证, 从而作为熔体团簇结构假 设的必要基础条件。在此基础上得到如图 1 所示的 $\mathrm{Na}_{2} \mathrm{O}-\mathrm{Al}_{2} \mathrm{O}_{3}-\mathrm{SiO}_{2}$ 三元体系熔体团簇结构。

\section{2 计算方法}

本文使用 Materials Studio 5.5 软件 ${ }^{22}$ 搭建不同 成分的 $\mathrm{Na}_{2} \mathrm{O}-\mathrm{Al}_{2} \mathrm{O}_{3}-\mathrm{SiO}_{2}$ 三元系团簇模型, 运用半 经验的 VAMP (Vienna Ab initio Molecular-dynamics Package) 模块对其进行结构优化和热力学计算。计 算参数设置为: 哈密顿函数选择 $\mathrm{MNDO} / \mathrm{d}^{23,24}$, 收 玫判据设为 $16.747 \mathrm{~kJ} \cdot \mathrm{mol}^{-1} \cdot \mathrm{nm}^{-1}$, 算法为 HartreeFock (RHF) 自洽分子轨道理论方法, 自洽场(SCF) 误差选择 $1.0 \times 10^{-5}$, 温度设置为 $100-2000 \mathrm{~K}$, 步 长为 $25 \mathrm{~K}$, 计算结果为熵、焓、热容在不同温度 下的值。计算结果将用于计算不同温度下该三元 体系的混合自由能。

\section{3 计算结果与讨论}

\section{1 $\mathrm{Na}_{2} \mathrm{O}-\mathrm{Al}_{2} \mathrm{O}_{3}-\mathrm{SiO}_{2}$ 三元系团簇基元混合自由能}

$\mathrm{Na}_{2} \mathrm{O}-\mathrm{Al}_{2} \mathrm{O}_{3}-\mathrm{SiO}_{2}$ 三元系团簇基元的熵、热容和 焓值可由计算结果文件中直接给出。团簇基元的 自由能 $G(T)$ 由下式来计算:

$$
G^{\prime}(T)=H_{(\mathrm{c})}(T)-T S_{(\mathrm{c})}(T)
$$

根据 VAMP 模块数据处理的需要, 若要求得各团 簇基元的自由能 $G(T)$, 需要对其进行以下修正:

$$
G(T)=G^{0}(298 \mathrm{~K})-G^{\prime}(298 \mathrm{~K})+G^{\prime}(T)
$$

其中: $G^{0}(298 \mathrm{~K})$ 为计算时输出文件中的生成热 (heat of formation)的值。 
$\oplus$

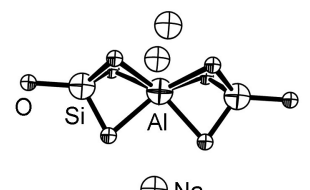

(a) $\mathrm{AlSi}_{2} \mathrm{O}_{8} \mathrm{Na}_{5}$

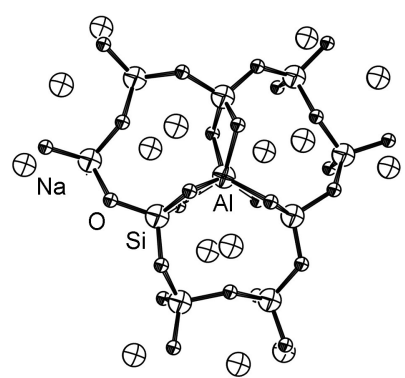

(e) $\mathrm{AlSi}_{9} \mathrm{O}_{27} \mathrm{Na}_{15}$

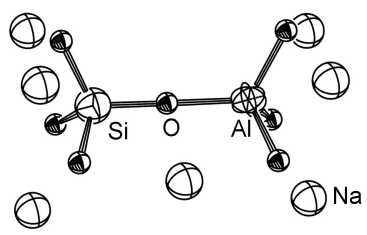

(h) $\mathrm{AlSiO}_{7} \mathrm{Na}_{7}$

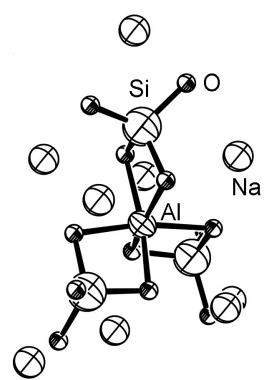

(b) $\mathrm{AlSi}_{3} \mathrm{O}_{12} \mathrm{Na}_{9}$

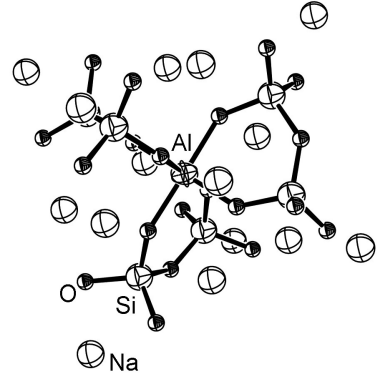

(c) $\mathrm{AlSi}_{6} \mathrm{O}_{21} \mathrm{Na}_{15}$

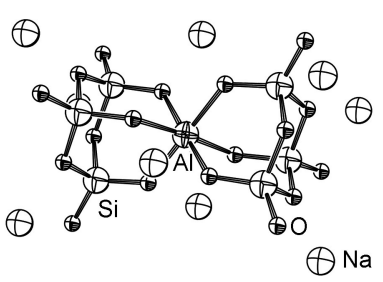

(d) $\mathrm{AlSi}_{6} \mathrm{O}_{18} \mathrm{Na}_{9}$

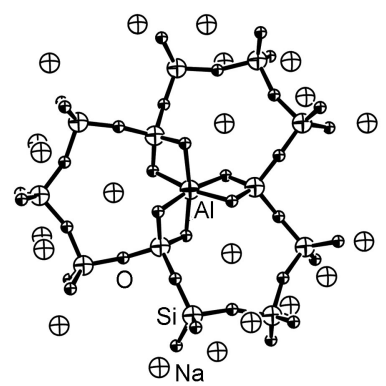

(f) $\mathrm{AlSi}_{12} \mathrm{O}_{36} \mathrm{Na}_{21}$

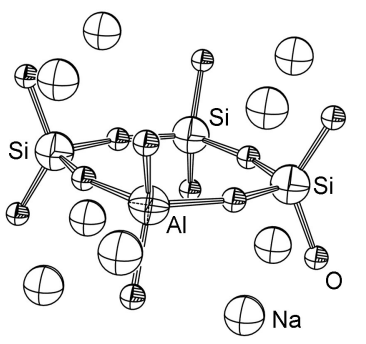

(i) $\mathrm{AlSi}_{3} \mathrm{O}_{12} \mathrm{Na}_{9}$

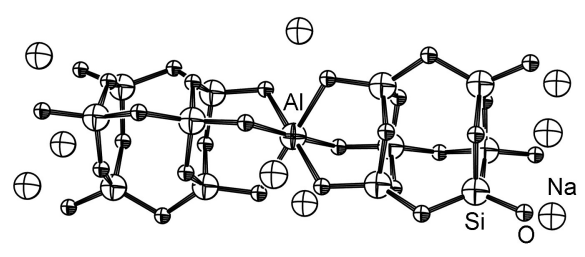

(g) $\mathrm{AlSi}_{12} \mathrm{O}_{30} \mathrm{Na}_{9}$

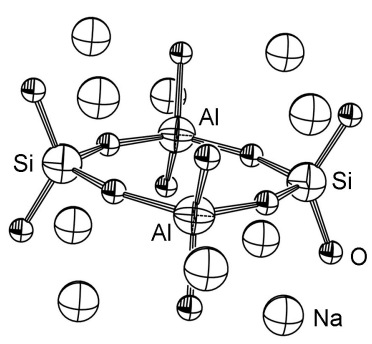

(j) $\mathrm{Al}_{2} \mathrm{Si}_{2} \mathrm{O}_{12} \mathrm{Na}_{10}$

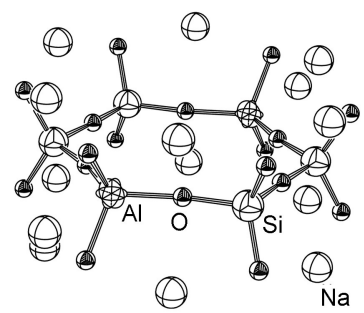

(k) $\mathrm{Al}_{2} \mathrm{Si}_{4} \mathrm{O}_{18} \mathrm{Na}_{14}$

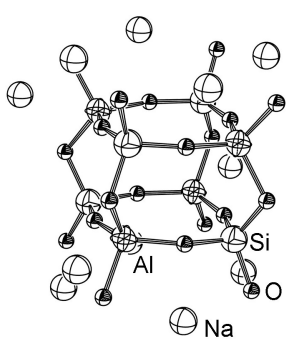

(o) $\mathrm{Al}_{2} \mathrm{Si}_{6} \mathrm{O}_{20} \mathrm{Na}_{10}$

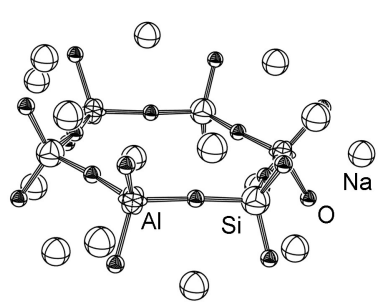

(I) $\mathrm{Al}_{3} \mathrm{Si}_{3} \mathrm{O}_{18} \mathrm{Na}_{15}$

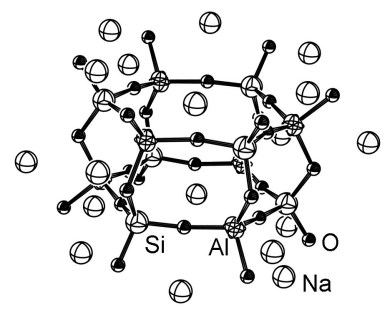

(p) $\mathrm{Al}_{6} \mathrm{Si}_{6} \mathrm{O}_{30} \mathrm{Na}_{18}$

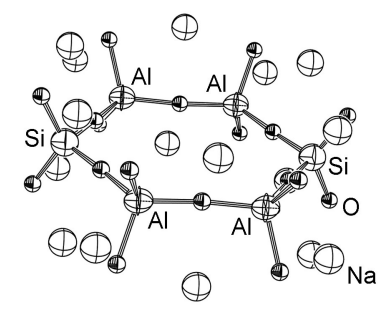

(m) $\mathrm{Al}_{4} \mathrm{Si}_{2} \mathrm{O}_{18} \mathrm{Na}_{16}$

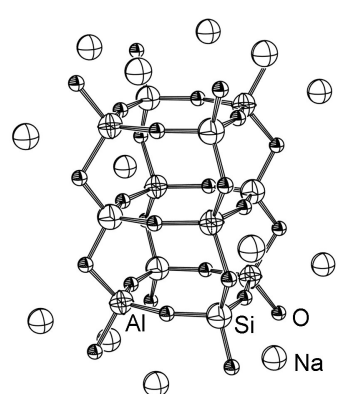

(q) $\mathrm{Al}_{6} \mathrm{Si}_{6} \mathrm{O}_{28} \mathrm{Na}_{14}$

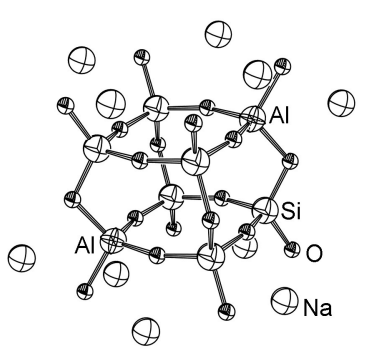

(n) $\mathrm{Al}_{4} \mathrm{Si}_{4} \mathrm{O}_{20} \mathrm{Na}_{12}$

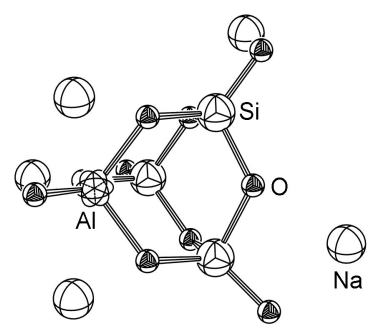

(r) $\mathrm{AlSi}_{3} \mathrm{O}_{10} \mathrm{Na}_{5}$

图 $1 \mathrm{Na}_{2} \mathrm{O}-\mathrm{Al}_{2} \mathrm{O}_{3}-\mathrm{SiO}_{2}$ 三元系团簇基元的构型图

Fig.1 Diagrams of model clusters of the $\mathrm{Na}_{2} \mathrm{O}-\mathrm{Al}_{2} \mathrm{O}_{3}-\mathrm{SiO}_{2}$ ternary system

Element labels are all the same as in the diagram of $\mathrm{AlSi}_{2} \mathrm{O}_{8} \mathrm{Na}_{5}$ (a). 
由半经验方法的计算结果得到不同温度下同 一团簇基元的熵、焓和热容, 再通过公式(1)和(2) 得出自由能。 $\mathrm{AlSi}_{2} \mathrm{O}_{8} \mathrm{Na}_{5}$ 团簇基元的熵、焓、热 容、自由能与温度的关系图见图 2。结构不同的硅 酸盐团簇, 它们的熵、焓、热容和自由能随温度 的变化规律是相同的, 其中熵、焓和热容随着温 度升高而有不同程度的增加, 自由能随着温度的 升高而减小。

为计算 $\mathrm{Na}_{2} \mathrm{O}-\mathrm{Al}_{2} \mathrm{O}_{3}-\mathrm{SiO}_{2}$ 三元系团簇基元在高 温下某一温度的混合自由能, 从 100 到 $2000 \mathrm{~K}$ 中 选取了硅酸盐熔体中常用的可用于比较的三个温 度点, 分别是 $1475 、 1875$ 和 $2000 \mathrm{~K}$ 。为了确定纯 物质 $\mathrm{Na}_{2} \mathrm{O} 、 \mathrm{Al}_{2} \mathrm{O}_{3}$ 和 $\mathrm{SiO}_{2}$ 在 $1473 、 1873$ 和 $2000 \mathrm{~K}$ 的自由能, 从 $\mathrm{Na}_{2} \mathrm{O} 、 \mathrm{SiO}_{2}$ 和 $\mathrm{Al}_{2} \mathrm{O}_{3}$ 的超晶胞中提取
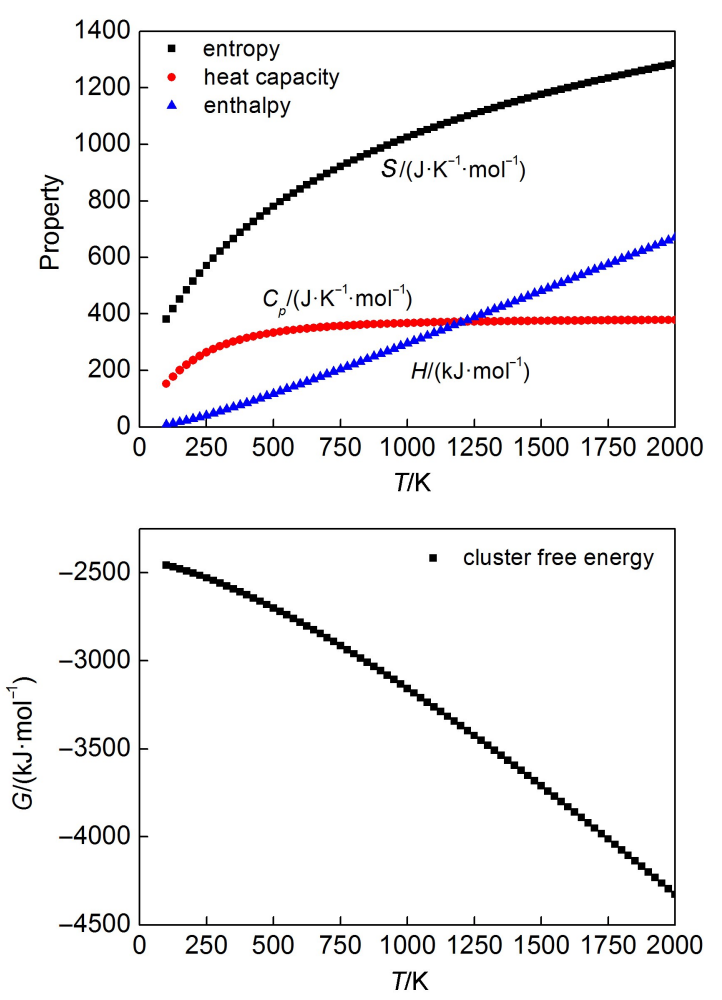

图 $2 \mathrm{AlSi}_{2} \mathrm{O}_{8} \mathrm{Na}_{5}$ 团簇基元(图 1(a))的熵、焓、热容、 自由能与温度的关系图

Fig.2 Temperatue dependent entropy, enthalpy, heat capacity, free energy of $\mathrm{AlSi}_{2} \mathrm{O}_{8} \mathrm{Na}_{5}$ cluster in Fig.1(a)
不同原子数的 $\mathrm{Na}_{2} \mathrm{O} 、 \mathrm{SiO}_{2}$ 和 $\mathrm{Al}_{2} \mathrm{O}_{3}$ 团簇基元, 结构 优化后用半经验方法 $\mathrm{MNDO} / \mathrm{d}$ 计算并将团簇基元 的自由能归一化为单位摩尔分数的 $\mathrm{Na}_{2} \mathrm{O} 、 \mathrm{SiO}_{2}$ 和 $\mathrm{Al}_{2} \mathrm{O}_{3}$, 归一化的自由能分别用 $G_{\text {min (N(N2, O) }} 、 G_{\text {min }\left(\mathrm{Si}_{2}\right)}$ 和 $G_{\text {mini }\left(\mathrm{Al}_{2} \mathrm{O}_{3}\right.}$ 表示。选择 $\mathrm{Na}_{2} \mathrm{O} 、 \mathrm{Al}_{2} \mathrm{O}_{3}$ 和 $\mathrm{SiO}_{2}$ 团簇基元自 由能的相对低点分别记为 $\mathrm{Na}_{2} \mathrm{O} 、 \mathrm{Al}_{2} \mathrm{O}_{3}$ 和 $\mathrm{SiO}_{2}$ 的稳 定能级。并假设团簇基元的能级分布满足准连续 近似, 根据统计热力学波尔兹曼分布定律, 通过 式(3)将团簇基元的自由能转化为体系的平均自由 能。

$$
\Delta G_{\mathrm{sys}}=\Delta G_{\min }-R T \ln \frac{1-\exp \left(\Delta G_{\min } / R T\right)}{-\Delta G_{\min } / R T}
$$

上式中 $\Delta G_{\min }$ 和 $\Delta G_{\mathrm{sys}}$ 分别为团簇基元和体系的摩 尔自由能, 计算结果见表 1 。从表 1 中可以发现, 纯物质 $\mathrm{Na}_{2} \mathrm{O} 、 \mathrm{Al}_{2} \mathrm{O}_{3}$ 和 $\mathrm{SiO}_{2}$ 体系混合自由能的计算 值要比实验值 ${ }^{25}$ 普遍偏高, 而且同一纯物质不同温 度的计算值与实验值之间的差值几乎一样。说明 目前采用 VAMP 模块计算物质的绝对能量存在系 统误差。熔体的混合自由能是一个相对能量值, 公式(4)中的各个项都是用同样的计算方法计算得 出, 系统误差在计算过程中会被抵消, 对最后结 果影响有限。

若以 $m \mathrm{Na}_{2} \mathrm{O} \cdot n \mathrm{Al}_{2} \mathrm{O}_{3} \cdot p \mathrm{SiO}_{2}$ 统一表示 $\mathrm{Na}_{2} \mathrm{O}-$ $\mathrm{Al}_{2} \mathrm{O}_{3}-\mathrm{SiO}_{2}$ 三元系团簇基元的组成(其中 $m 、 n$ 和 $p$ 分别为 $\mathrm{Na}_{2} \mathrm{O} 、 \mathrm{Al}_{2} \mathrm{O}_{3}$ 和 $\mathrm{SiO}_{2}$ 的摩尔分数), 则 $\mathrm{Na}_{2} \mathrm{O}-$ $\mathrm{Al}_{2} \mathrm{O}_{3}-\mathrm{SiO}_{2}$ 团簇基元的混合摩尔自由能可通过公式 (4)归一化求得。 $\mathrm{Na}_{2} \mathrm{O}-\mathrm{Al}_{2} \mathrm{O}_{3}-\mathrm{SiO}_{2}$ 团簇基元的混合 摩尔自由能计算如图3 所示。

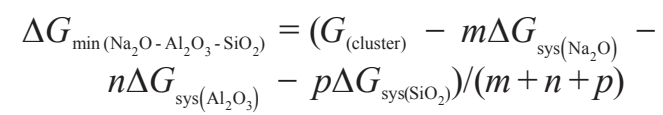

本工作是基于团簇模型作为熔体结构的基本 假设。从 $\mathrm{Na}_{2} \mathrm{O}-\mathrm{Al}_{2} \mathrm{O}_{3}-\mathrm{SiO}_{2}$ 三元体系相图 ${ }^{26}$ (见图 3) 可 知, 编号为 $(g) 、(h) 、(j) 、(1)$ 和 $(m)$ 的团簇模型所在 成分点的材料在 $1473 \mathrm{~K}$ 下并未熔解, 因此会表现 较大差异。但这五个团簇结构的成分点材料在 1873 和 $2000 \mathrm{~K}$ 高温下皆处于熔体状态, 因此它们

表 1 归一化后的 $\mathrm{Li}_{2} \mathrm{O} 、 \mathrm{Na}_{2} \mathrm{O}$ 和 $\mathrm{SiO}_{2}$ 团簇的体系自由能

\begin{tabular}{|c|c|c|c|c|c|c|}
\hline \multirow{2}{*}{$T / \mathrm{K}$} & \multicolumn{2}{|c|}{$\Delta G_{\mathrm{sy}\left(\mathrm{Na}_{2} \mathrm{O}\right)} /\left(\mathrm{kJ} \cdot \mathrm{mol}^{-1}\right)$} & \multicolumn{2}{|c|}{$\Delta G_{\mathrm{sy}\left(\mathrm{Al}_{2} \mathrm{O}_{3}\right)} /\left(\mathrm{kJ} \cdot \mathrm{mol}^{-1}\right)$} & \multicolumn{2}{|c|}{$\Delta G_{\mathrm{sys}\left(\mathrm{SiO}_{2}\right)} /\left(\mathrm{kJ} \cdot \mathrm{mol}^{-1}\right)$} \\
\hline & calculation value & actual value $\mathrm{e}^{25}$ & calculation value & actual value ${ }^{25}$ & calculation value & actual value $^{25}$ \\
\hline 1473 & -430.363 & -632.060 & -1428.236 & -1875.880 & -750.809 & -1044.754 \\
\hline 1873 & -541.333 & -740.850 & -1555.526 & -1974.418 & -838.761 & -1106.598 \\
\hline 2000 & -571.707 & -776.750 & -1597.550 & -2008.192 & -870.512 & -1127.219 \\
\hline
\end{tabular}

Table 1 Normalized free energy of $\mathrm{Li}_{2} \mathrm{O}, \mathrm{Na}_{2} \mathrm{O}$, and $\mathrm{SiO}_{2}$ clusters 


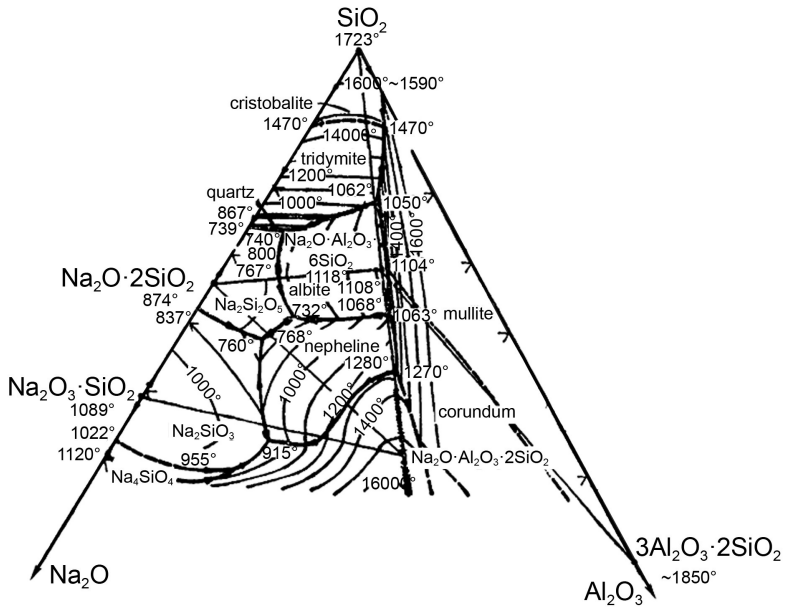

图 $3 \mathrm{Na}_{2} \mathrm{O}-\mathrm{Al}_{2} \mathrm{O}_{3}-\mathrm{SiO}_{2}$ 三元相图 ${ }^{26}$

Fig.3 Phasc diagram of $\mathrm{Na}_{2} \mathrm{O}-\mathrm{Al}_{2} \mathrm{O}_{3}-\mathrm{SiO}_{2}{ }^{26}$

适用于 1873 和 $2000 \mathrm{~K}$ 下的模拟计算。

团簇基元混合自由能的计算值如图 4、图 5 和 图6所示呈散点分布。通过将各成分的最低点篮选 拟合出一个相对光滑且无奇点的光滑曲面(如图 4 中实线网格曲面), 并称该曲面为团簇基元最低能 量前沿(CULEF), 这意味着该体系中不会存在一个 团簇基元的混合自由能低于该曲面。处在 CULEF 曲面附近的团簇基元混合自由能较低, 这些团簇 基元在熔体中将会较稳定存在并且其分布权重也 大。又由计算过程可知, 由于团簇基元的混合自 由能是通过差减得到的相对能量, 有效地降低了 因计算方法而带来的系统误差，因而更具有实际 参考价值。

从以上内容可知，团簇基元 $(\mathrm{f}) 、(\mathrm{~g}) 、(\mathrm{~h})$ 、

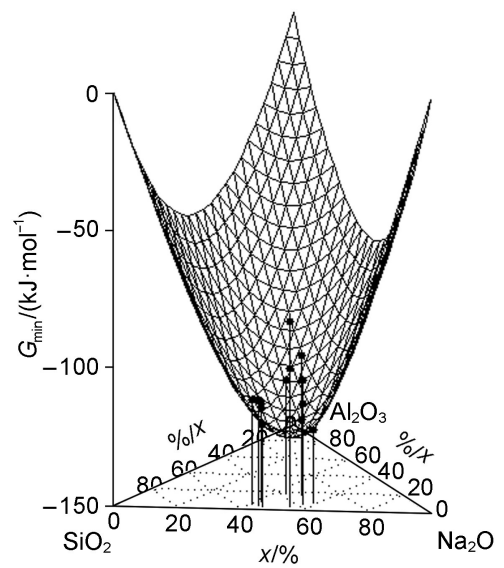

图 $4 \mathrm{Na}_{2} \mathrm{O}-\mathrm{Al}_{2} \mathrm{O}_{3}-\mathrm{SiO}_{2}$ 团簇基元在 $1473 \mathrm{~K}$ 的混合 自由能和其最低能量前沿

Fig.4 Mixing free energy of $\mathrm{Na}_{2} \mathrm{O}-\mathrm{Al}_{2} \mathrm{O}_{3}-\mathrm{SiO}_{2}$ cluster and cluster unit lowest energy frontier CULEF at $1473 \mathrm{~K}$

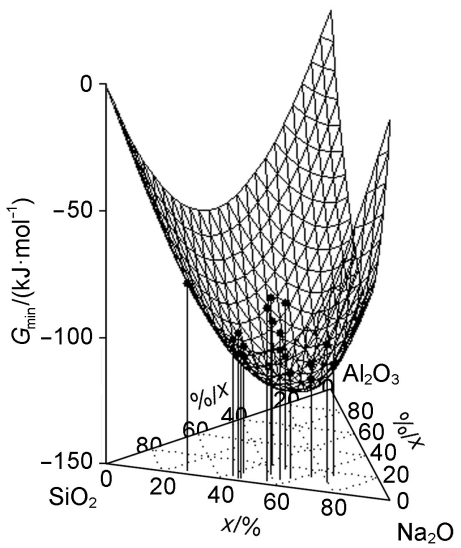

图 $5 \mathrm{Na}_{2} \mathrm{O}-\mathrm{Al}_{2} \mathrm{O}_{3}-\mathrm{SiO}_{2}$ 团簇基元在 $1873 \mathrm{~K}$ 的混合 自由能和其最低能量前沿

Fig.5 Mixing free energy of $\mathrm{Na}_{2} \mathrm{O}-\mathrm{Al}_{2} \mathrm{O}_{3}-\mathrm{SiO}_{2}$ cluster and cluster unit lowest energy frontier CULEF at $1873 \mathrm{~K}$

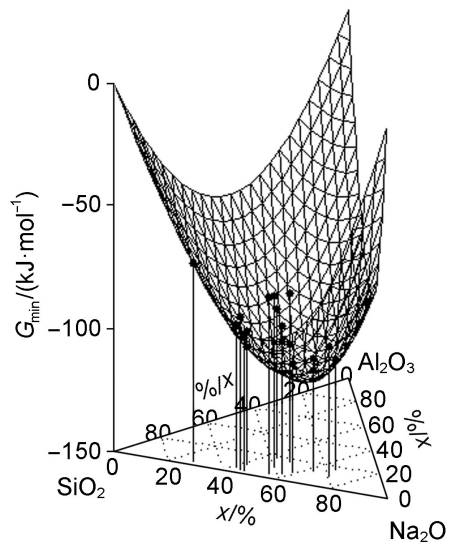

图 $6 \mathrm{Na}_{2} \mathrm{O}-\mathrm{Al}_{2} \mathrm{O}_{3}-\mathrm{SiO}_{2}$ 团簇基元在 $2000 \mathrm{~K}$ 的混合 自由能和其最低能量前沿

Fig.6 Mixing free energy of $\mathrm{Na}_{2} \mathrm{O}-\mathrm{Al}_{2} \mathrm{O}_{3}-\mathrm{SiO}_{2}$ cluster and cluster unit lowest energy frontier CULEF at $2000 \mathrm{~K}$

(j)、(k)、(l)、(m)、(r) 是最接近最低能量前沿 CULEF 曲面的团簇模型，因此它们是在高温下各 自的成分点上的分布权重较大的团簇结构。而且 我们得到了稳定团簇结构的同时，也得到了该三 元体系的团簇结构在不同温度和成分下的最低混 合自由能值。

\section{2 $\mathrm{Na}_{2} \mathrm{O}-\mathrm{Al}_{2} \mathrm{O}_{3}-\mathrm{SiO}_{2}$ 三元体系平均混合自由能}

在较高的温度下, 团簇基元的键长和键角会 在一定范围内变化，这导致团簇基元的混合自由 能发生连续变化。由此可知, 团簇基元混合自由 能的能级分布依然满足准连续近似, 同样通过(3) 式, 可由最低能量前沿的混合自由能求得 $\mathrm{Na}_{2} \mathrm{O}$ $\mathrm{Al}_{2} \mathrm{O}_{3}-\mathrm{SiO}_{2}$ 三元体系的平均混合自由能(如图 7 中的 曲面)。 $\mathrm{Na}_{2} \mathrm{O}-\mathrm{Al}_{2} \mathrm{O}_{3}-\mathrm{SiO}_{2}$ 三元体系的平均混合自由 能与 HSC chemistry 热学数据 ${ }^{27}$ 进行对比, 结果见 

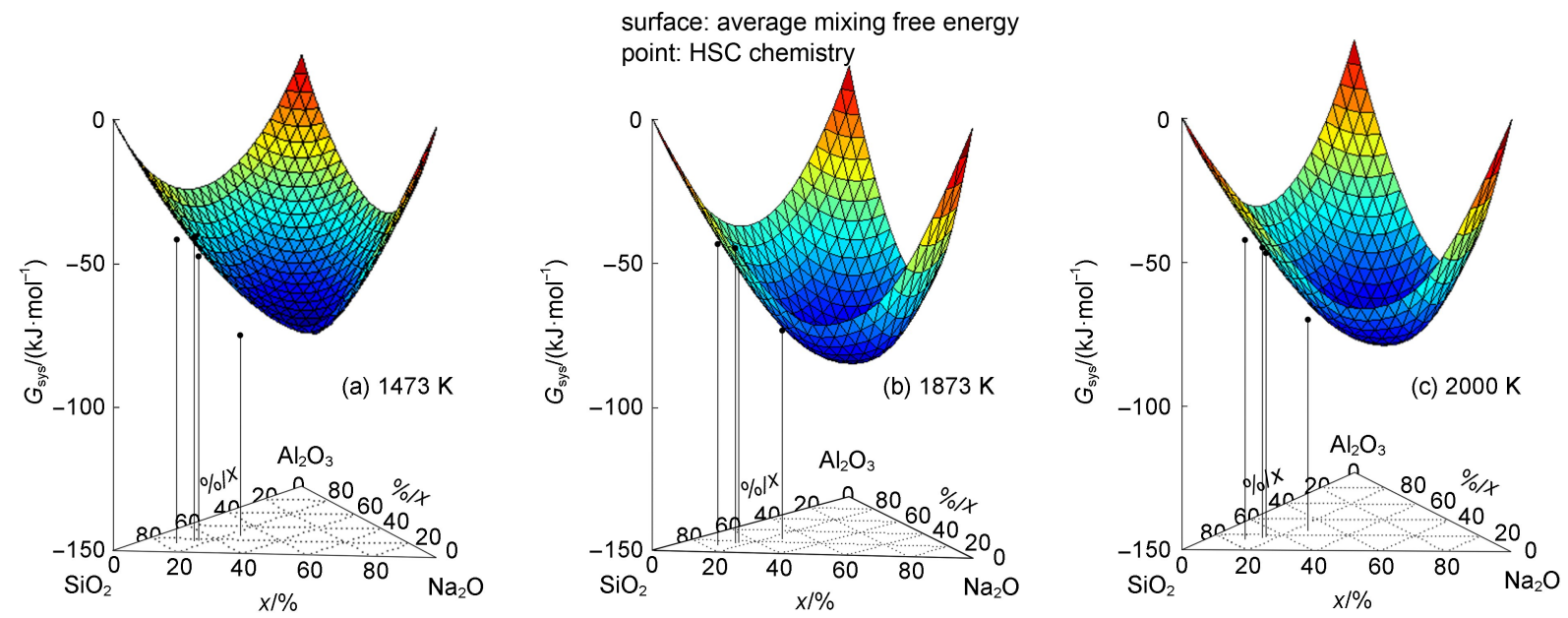

图 $7 \mathrm{Na}_{2} \mathrm{O}-\mathrm{Al}_{2} \mathrm{O}_{3}-\mathrm{SiO}_{2}$ 三元系在 $1473 、 1873$ 和 $2000 \mathrm{~K}$ 的平均混合自由能和 $\mathrm{HSC}$ chemistry 热学数据的比较

Fig.7 Comparison of average mixing free energy of $\mathrm{Na}_{2} \mathrm{O}-\mathrm{Al}_{2} \mathrm{O}_{3}-\mathrm{SiO}_{2}$ system with $\mathrm{HSC}$ chemistry data calculated at 1473, 1873, and $2000 \mathrm{~K}$

图7。

从图 7可以看出平均混合自由能和HSC chemistry 热学数据有一定的误差, 误差小于 $10 \%$ 。1473 K 下 $\mathrm{NaAlSiO}_{4}$ 通过本工作计算得出的平均混合摩尔 自由能与 HSC 计算值有较大的误差。原因是 NaAl$\mathrm{SiO}_{4}$ 在 $1473 \mathrm{~K}$ 下仍未熔解 (见图 3), 晶体状态下的 $\mathrm{NaAlSiO}_{4}$ 的平均混合自由能显然比以计算熔体的 方法模拟计算得到的平均混合自由能低。相反, 在 $1473 \mathrm{~K}$ 处于熔体状态的其他三个成分点计算得 出的结果与 HSC 计算值吻合较好, 并且在 1873 和 $2000 \mathrm{~K}$ 下处于熔体状态的 $\mathrm{NaAlSiO}_{4}$ 的 $\mathrm{HSC}$ 计算值 依然与本工作模拟计算得出的平均混合摩尔自由 能吻合较好。这种差异恰恰验证了本工作模拟计 算方法的合理性。

\section{4 结 论}

通过分子模拟半经验量化计算, 利用 Materials Studio 5.5 计算软件中半经验的 MNDO/d 计算方 法计算得出 $\mathrm{Na}_{2} \mathrm{O}-\mathrm{Al}_{2} \mathrm{O}_{3}-\mathrm{SiO}_{2}$ 三元系的不同团簇基 的熵、焓、热容和自由能随温度变化的能量曲 线。进而计算得出 $\mathrm{Na}_{2} \mathrm{O}-\mathrm{Al}_{2} \mathrm{O}_{3}-\mathrm{SiO}_{2}$ 三元系不同的 团簇基元的混合自由能，从而推导出该成分点在 体系中的 1473、1873 和 $2000 \mathrm{~K}$ 的平均混合自由 能。VAMP模块的计算方法仍存在系统误差, 但是 熔体的混合自由能是一个相对值, 公式中的各个 项都是用同样的计算方法计算得出, 系统误差在 这样的计算过程中会被消除掉, 对混合自由能值 没有太大的影响。 $\mathrm{Na}_{2} \mathrm{O}-\mathrm{Al}_{2} \mathrm{O}_{3}-\mathrm{SiO}_{2}$ 三元体系的平均
混合自由能计算结果与 HSC chemistry 数据库的热 学数据吻合较好, 表明了 $\mathrm{Na}_{2} \mathrm{O}-\mathrm{Al}_{2} \mathrm{O}_{3}-\mathrm{SiO}_{2}$ 三元体 系的热力学性能不仅与硅氧四面体的种类有关, 而且与硅氧四面体的近邻连接情况和铝的配位数 有关, 即与硅酸盐的精细结构密切相关。搭建硅 酸盐团簇模型需要预先了解硅酸盐熔融后的结构 形态, 掌握其高温特性。本研究工作表明团簇模 型可以描述熔体中的微观结构及获取相关的热力 学性能。

\section{References}

(1) Ma, X. C.; Yu, C. M.; Cheng, G. G. Journal of Iron and Steel Research 2011, 5, 3. [马小春, 于春梅, 成国光. 钢铁研究学 报, 2011, 5, 3.] doi: 10.3969/j.issn.1001-7208.2011.02.010

(2) Zhang, J. Journal of Beijing University of Iron and Steel Technology 1988, 10, 412. [张鉴. 北京钢铁学院学报, 1988, 10, 412.] doi: 10.13374/j.issn1001-053x.1988.04.023

(3) Lü, N. N.; Yu, J. K.; Su, C.; Wang, H. Z. Journal of Northeastern University Natural Science 2013, 34 (12), 1743. [吕宁宁, 于景坤, 苏 畅, 王洪章. 东北大学学报(自然科学 版), 2013, 34 (12), 1743.]

(4) Mysen, B. O.; Lucier, A.; Cody, G. D. American Mineralogist 2003, 88 (11-12), 1668. doi: 10.2138/am-2003-11-1206

(5) Lin, P.; Pelton, A. Metallurgical Transactions B 1979, 10 (4), 667. doi: 10.1007/BF02662569

(6) Masson, C. Journal of the American Ceramic Society 1968, 51 (3), 134. doi: 10.1111/jace.1968.51.issue-3

(7) Ottonello, G. Journal of Non-Crystalline Solids 2001, 282 (1), 72. doi: $10.1016 / \mathrm{S} 0022-3093(01) 00330-1$

(8) Zaitsev, A. I.; Arutyunyan, N. A.; Shaposhnikov, N. G.; Zaitseva, N. E.; Burtsev, V. T. Russ. J. Phys. Chem. 2006, 80 (3), 335. doi: 10.1134/S0036024406030058 
(9) Glibin, V. P.; King, P. L. Calphad-Comput. Coupling Ph. Diagrams Thermochem. 2015, 49, 19. doi: 10.1016/j. calphad.2015.02.001

(10) Neuville, D. R.; Cormier, L.; Massiot, D. Chemical Geology 2006, 229 (1), 173. doi: 10.1016/j.chemgeo.2006.01.019

(11) Yang, X. Y.; Vantomme, A.; Xiao, F. S.; Su, B. L.Catalysis Today 2007, 128 (3), 123. doi: 10.1016/j.cattod.2007.07.017

(12) Liu, Q.; You, J. L.; Wang, Y. Y.; Wang, C. Y.; Wang, J.; Liu, X. W. Spectroscopy and Spectral Analysis 2013, 10, 31. [刘 钦, 尤静林, 王媛媛, 王晨阳, 王 静, 刘晓伟. 光谱学与光谱分 析, 2013, 10, 31.] doi: 10.3964/j.issn.1000-0593(2013)102705-06

(13) Wang, Y. Y.; You, J. L.; Jiang, G. C. Chinese Journal of Inorganic Chemistry 2008, 24 (5), 765. [王媛媛, 尤静林, 蒋国 昌. 无机化学学报, 2008, 24 (5), 765.]

(14) Wang, J.; You, J. L.; Wang, Y. Y. Journal of Synthetic Crystals 2013, 3, 4. [王 静, 尤静林, 王媛媛. 人工晶体学报, 2013, 3, 4.]

(15) Wu, J.; Stebbins, J. F. Journal of Non-Crystalline Solids 2010, 356 (41), 2097. doi: 10.1016/j.jnoncrysol.2010.08.015

(16) You, J.; Jiang, G.; Hou, H. Isomorphic Representations of Hyperfine Structure of Binary Silicates by Interior Stress, Vibrational Wavenumber and Special Fractional Dimension. In Journal of Physics: Conference Series; IOP Publishing Ltd.: Singapore 2006; p 25.

(17) You, J. L.; Jiang, G. C.; Hou, H. Y. Journal of Raman Spectroscopy 2005, 36 (3), 237. doi: 10.1002/jrs. 1287

(18) Wang, W.; You, J. L. Thermodynamic Calculation for $\mathrm{LiO}_{2}-$ $\mathrm{SiO}_{2}$ and $\mathrm{Na}_{2} \mathrm{O}-\mathrm{SiO}_{2}$ Binary Melt Based on Cluster Structure
Model of Molten Silicates. Shanghai University, Shanghai, 2009. [王 威, 尤静林. 基于团簇结构模型的 $\mathrm{Li}_{2} \mathrm{O}-\mathrm{SiO}_{2}$ 、 $\mathrm{Na}_{2} \mathrm{O}-\mathrm{SiO}_{2}$ 二元系熔体的热力学性质计算 $[\mathrm{D}]$. 上海: 上海大 学, 2009.]

(19) Park, S. Y.; Lee, S. K. Geochimica Et Cosmochimica Acta 2014, 147, 26. doi: 10.1016/j.gca.2014.10.019

(20) Neuville, D.; Cormier, L.; Ligny, D. American Mineralogist 2008, 93 (1), 228. doi: 10.2138/am.2008.2646

(21) You, J. L.; Wang, Y. Y.; Jiang, G. C.; Xie, J.; Yu, X. H.; Mo, X. X. Quantum Chemistry ab initio Calculation Study of Structure and Raman Spectra of Aluminosilicates. In Xxii International Conference on Raman Spectroscopy, Proceedings of the AIP Conference, Boston, America, Aug 8 13, 2010; Champion, P. M., Ziegler, L. D. Eds.; Elsevier: USA, 2010.

(22) Segall, M.; Linda, P.; Probert, M. Materials Studio, Version 5.5; Accelrys: American, 2010.

(23) Thiel, W.; Voityuk, A. A. Theoretica Chimica Acta 1992, 81 (6), 391. doi: 10.1007/BF01134863

(24) Thiel, W.; Voityuk, A. A. The Journal of Physical Chemistry 1996, 100 (2), 616. doi: 10.1021/jp952148o

(25) Farnan, I.; Stebbins, J. Journal of the American Chemical Society 1990, 112 (1), 32. doi: 10.1021/ja00157a008

(26) Rui, L. Physical Chemistry of Inorganic Materials; Chinese Construction Industry Press: Beijing, 1986; p 194. [瑞伦. 无 机材料物理化学. 北京: 中国建筑工业出版社, 1986: 194.]

(27) Roine, A. HSC Chemistry, Ver 6.1; OutokumpuOy: Finland, 2002. 